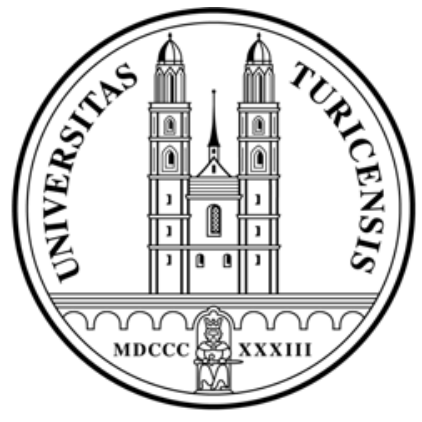

Institute for Empirical Research in Economics

University of Zurich

Working Paper Series

ISSN 1424-0459

Working Paper No. 303

\title{
Providing Public Goods in the Absence of Strong Institutions
}

Anke Gerber and Philipp C. Wichardt

February 2008 


\title{
Providing Public Goods in the Absence of Strong Institutions*
}

\author{
Anke Gerber ${ }^{\dagger}$ \\ Department of Economics, University of Hamburg \\ Philipp C. Wichardt \\ Department of Economics, University of Bonn
}

This Version: January 14, 2008

\begin{abstract}
This paper proposes a simple two-stage mechanism to establish positive contributions to public goods in the absence of powerful institutions to provide the public good and to sanction free-riders. In this mechanism players commit to the public good by paying a deposit prior to the contribution stage. If there is universal commitment, deposits are immediately refunded whenever a player contributes her specified share to the public good. If there is no universal commitment, all deposits are refunded and the standard game is played. For suitable deposits, prior commitment and full ex post contributions are supported as a strict subgame perfect Nash equilibrium for the resulting game. As the mechanism obviates the need for any ex post prosecution of free-riders, it is particularly suited for situations where players do not submit to a common authority as in the case of international agreements.
\end{abstract}

Key words: Public Goods, Cooperation, Institutions, Climate-Change Treaties JEL code: C72, D61

*Acknowledgements: We are grateful to Sylvain Béal and Patrick Schmitz for helpful comments and discussions. Philipp Wichardt gratefully acknowledges financial support from the German Research Foundation (DFG) through GRK 629 and SFB/TR 15 at the University of Bonn.

${ }^{\dagger}$ Postal address for correspondence: Department of Economics, University of Hamburg, Von-Melle-Park 5, D-20146 Hamburg, Germany; e-mail: anke.gerber@wiso.uni-hamburg.de.

${ }_{\ddagger}^{\ddagger}$ e-mail: philipp.wichardt@uni-bonn.de 


\section{Introduction}

One of the most prominent examples for the failure of markets concerns the provision of public goods. The conflict of interest between the socially desirable and individually optimal contribution to the public good commonly prevents the implementation of Pareto optimal solutions — not only theoretically but also empirically (e.g. Fehr and Gächter, 2000). Due to the often immense welfare gains at stake (e.g. clean water/air), the question of how to establish high contribution rates in public goods games is a key issue in economic policy.

An "easy" way out of such social dilemmas is the introduction of sanctioning institutions that enforce contributions to public goods (e.g. a reliable jurisdiction). Already casual evidence suggests that, once individual deviations from previously agreed contribution rates can be appropriately punished, many public goods can be - and indeed are - established at a level close to the social optimum (e.g. public transport, health care, quiet sleeping hours at night). Evidence from laboratory experiments further supports this observation (e.g. Falkinger et al., 2000; Fehr and Gächter, 2000). In fact, more recent studies even show that individuals, when facing the choice between a social environment with and without sanctioning possibilities, learn to choose the environment with sanctioning (Gürerk et al., 2006; see also Kosfeld et al., 2006). Moreover, upon being placed in the desired environment, most players indeed fully contribute to the public good and punish free-riders (Gürerk et al., 2006).

A sanctioning mechanism, however, requires two things: Firstly, it requires the presence of an institution with the power to enforce potential sanctions; and secondly, it requires a social planner who is sufficiently well informed about the agents' preferences so that she can impose effective sanctions. Under certain conditions the informational requirement can be relaxed and the planner can design a game form which, if played by the agents, yields in equilibrium the outcome prescribed by a social choice function for any profile of preferences. There is a large literature on how to implement a social choice function in this way and we do not even attempt to provide a complete survey here. The well-known ClarkeGroves mechanisms (Clarke, 1971, and Groves, 1973) implement an efficient level of a public good in a dominant strategy equilibrium but the mechanisms are not budget-balanced and do not yield a Pareto efficient allocation in general. 
Indeed Green and Laffont (1979) show that there is a fundamental conflict between efficiency and incentive compatibility whenever implementation has to be in dominant strategies. If instead one considers implementation in subgame perfect equilibrium, though, more positive results can be obtained. In fact, almost any social choice rule can be implemented by subgame perfect equilibrium (Moore and Repullo, 1988; Palfrey and Srivastava, 1991; Jackson, 1992). However, the required mechanisms often are very complex and, hence, of little use for practical purposes. Several authors have therefore explored simple mechanisms (see, e.g., Bagnoli and Lipman, 1989; Jackson and Moulin, 1992; Varian, 1994).

What is common to all these mechanisms is that they still require the existence of a strong institution to implement transfers between the agents. Moreover, it is the institution or the social planner himself who finally provides the public good. Clearly, these conditions are likely to be satisfied in case of local public goods, where governmental institutions exist to back up the enforcement and where the public good is directly provided by the government (education, streetlight, national security etc.). Yet, in a more and more globalised world, an increasing number of public goods does not belong to this category. Most prominent among them are public goods related to environmental issues like global warming, which have received a lot of attention in the recent economic literature (cf. Carraro, 1999; Bosello, Buchner, and Carraro, 2003; Dutta and Radner, 2004). Here, the public good "clean air" has to be provided in a decentralized way as each country has to take measures in order to reduce greenhouse gas emissions. At the same time effective sanctioning of free-riders is difficult to establish since there is no institution like a world government which can punish sovereign countries. Hence, it is not surprising that we observe a major compliance problem with climate-change treaties like the Kyoto Protocol.

In the present paper, we take up this issue and propose a simple mechanism aimed to establish the provision of a public good in cases where effective ex post sanctioning is difficult, if not impossible to enforce, and where the public good has to be provided in a decentralized way by the agents themselves. For our analysis, we take as given some agreement on individual contributions to the public good; i.e. we are not concerned with the agents' incentives to actually reach such an agreement but only with its eventual implementation. The considered agreement 
can, for example, be thought of as the result of some unspecified cooperative bargaining process among the agents. It may be characterized by a number of desirable properties like efficiency, individual rationality, equity etc. Like the Kyoto protocol, however, the agreement is not enforceable due to the absence of sufficiently strong institutions. Hence, it will be implemented only if all agents find it in their self-interest to do so.

The idea of our mechanism is to allow players to take an action, prior to the contribution stage, which renders it a dominant strategy to comply with the agreement. More specifically, we consider a 2-stage modification of a general public goods game. In stage 1, players can choose to pay a deposit to a neutral institution. If at the end of stage 1 everyone has paid a deposit, then in stage 2 the public goods game is played and deposits are refunded to those who contribute to the public good. If some player has not paid the deposit, all deposits are refunded (potentially deducting a small fee to sustain the institution administering deposits) and the standard public goods game is played in stage 2 . Obviously, universal commitment in the form of paying the deposit as well as full contributions to the public good now can be rationalised as a subgame perfect Nash equilibrium of the resulting game. Moreover, as players essentially execute their own punishment (pay the deposit), the neutral institution considered here only has to resist demands to repay forfeited deposits. This, however, appears far easier to enforce than collecting fines from free-riders ex post.

The rest of the paper is structured as follows. In Section 2, we introduce and analyse our mechanism. A discussion of the mechanism is provided in Section 3.

\section{The Mechanism}

We consider a standard public goods problem with $n$ players referred to as consumers. Each consumer $i, i=1, \ldots, n$, has a preference relation over consumption of a private and a public good. These preferences are represented by a utility function $U_{i}: \mathbb{R}_{+}^{2} \rightarrow \mathbb{R}$, where $U_{i}\left(x_{i}, z\right)$ is $i$ 's utility from consuming $x_{i}$ units of the private good and $z$ units of the public good. We assume that $U_{i}$ is strictly increasing: 
Assumption A: For all $i$, it holds that

$$
\frac{\partial U_{i}\left(x_{i}, z\right)}{\partial x_{i}}>0 \text { and } \quad \frac{\partial U_{i}\left(x_{i}, z\right)}{\partial z}>0
$$

Each consumer $i$ has an initial endowment $e_{i}>0$ of the private good while the initial level of the public good is zero. The public good is produced in a decentralized way, i.e. for all $i$ there exists a differentiable production function $f_{i}:\left[0, e_{i}\right] \rightarrow \mathbb{R}_{+}$with $f_{i}(0)=0, f_{i}^{\prime}>0$, and $f_{i}^{\prime \prime}<0$. If consumer $i$ contributes $c_{i} \in\left[0, e_{i}\right], i=1, \ldots, n$, the realized amount of the public good is

$$
z=F\left(\sum_{i=1}^{n} f_{i}\left(c_{i}\right)\right)
$$

for some differentiable function $F: \mathbb{R}_{+} \rightarrow \mathbb{R}_{+}$with $F^{\prime}>0$.

In the context of global warming, the $f_{i}$ can, for example, be thought of as the country's technology for reducing greenhouse gas emissions, while $F\left(\sum_{i} r_{i}\right)$ can be interpreted as the "quality" of the atmosphere if each country $i$ has reduced its greenhouse gas emissions by $r_{i}{ }^{1}$

If consumers contribute $c_{1}, \ldots, c_{n}$, consumer $i$ 's utility is given by:

$$
\pi_{i}\left(c_{1}, \ldots, c_{n}\right):=U_{i}\left(e_{i}-c_{i}, F\left(\sum_{j=1}^{n} f_{j}\left(c_{j}\right)\right)\right)
$$

Finally, in order to capture the essence of the public goods problem at issue, we assume that voluntary contributions are always strictly dominated.

Assumption B: For all $\left(c_{1}, \ldots, c_{n}\right) \in \times_{j=1}^{n}\left[0, e_{j}\right]$, it holds that

$$
\frac{\partial U_{i}\left(x_{i}, z\right)}{\partial x_{i}}>\frac{\partial U_{i}\left(x_{i}, z\right)}{\partial z} f_{i}^{\prime}\left(c_{i}\right) F^{\prime}\left(\sum_{j} f_{j}\left(c_{j}\right)\right)
$$

for all $x_{i}$ and for all $i=1, \ldots, n$, where $z=F\left(\sum_{j} f_{j}\left(c_{j}\right)\right)$.

Under Assumption B it follows that $\partial \pi_{i}\left(c_{1}, \ldots, c_{n}\right) / \partial c_{i}<0$ for all $i$ and all contribution profiles $\left(c_{1}, \ldots, c_{n}\right)$.

\footnotetext{
${ }^{1}$ Observe that the quality of the atmosphere need not be a linear function of the aggregate reduction in greenhouse gas emissions.
} 
A simple example for the class of problems considered here is the linear public goods problem, where $U_{i}\left(x_{i}, z\right)=x_{i}+b_{i} z, f_{i}\left(c_{i}\right)=c_{i} / a_{i}$ for some positive constants $a_{i}, b_{i}$ with $a_{i}>b_{i}$, and $F(r)=r$ for all $r \geq 0$. For this case, it is immediate to see that Assumptions A and B are satisfied.

The resulting public goods problem is modelled as an $n$-player game $P G$, where each player $i$ has a strategy set $S_{i}=\left[0, e_{i}\right]$ and payoff function $\pi_{i}$ : $\times_{j=1}^{n} S_{j} \rightarrow \mathbb{R}$. Under Assumption B the following Proposition is immediate:

Proposition 1 The strategy profile $c^{0}$ with $c_{i}^{0}=0$ for all $i$ is the unique Nash equilibrium for $P G$. Moreover, $c_{i}^{0}=0$ is a strictly dominant strategy for all $i$.

As, in general, the no contribution equilibrium in Proposition 1 does not lead to an efficient outcome, there is a conflict between individual incentives and aggregate welfare. The mechanism proposed below remedies this deficiency. More specifically, it is designed to implement an arbitrary contribution profile $\bar{c}=\left(\bar{c}_{1}, \ldots, \bar{c}_{n}\right) \in \times_{j=1}^{n}\left(0, e_{j}\right]$ that Pareto dominates the no contribution profile, i.e.

$$
\pi_{i}\left(\bar{c}_{1}, \ldots \bar{c}_{n}\right)>\pi_{i}(0, \ldots, 0) \text { for all } i=1, \ldots, n .
$$

As mentioned before, the vector of contributions $\bar{c}$ can, for example, be thought of as the result of some cooperative bargaining process among the players which cannot be enforced, e.g. due to a lack of strong institutions.

Beyond (1), no further assumptions on $\bar{c}$ are necessary. In particular, it is not required that $\bar{c}$ yields a Pareto efficient allocation. To implement $\bar{c}$ consider the following 2-stage modification of $P G$.

Stage 1 All players simultaneously choose to pay a deposit $d_{i} \in\left\{0, \bar{d}_{i}\right\}, \bar{d}_{i}>0$, to a neutral institution, e.g. a trustee. At the end of stage 1, the profile of all deposits paid, denoted by $d$, is revealed to the players. Thereafter, the game enters stage 2 .

Stage 2 The interaction in this stage depends on $d$ in the following way. If at the end of stage 1 we have $d_{i}=0$ for some $i$, then all deposits are refunded and in stage 2 the public goods game $P G$ is played. If, however, at the end of stage 1 we have $d_{i}=\bar{d}_{i}$ for all $i$, then in stage 2 a public goods game $P G^{*}$ is played for which payoffs are as follows: 


$$
\pi_{i}^{*}\left(c_{1}, \ldots, c_{n}\right)= \begin{cases}U_{i}\left(e_{i}-c_{i}-d_{i}, F\left(\sum_{j} f_{j}\left(c_{j}\right)\right)\right), & \text { if } c_{i} \neq \bar{c}_{i} \\ U_{i}\left(e_{i}-c_{i}, F\left(\sum_{j} f_{j}\left(c_{j}\right)\right)\right), & \text { if } c_{i}=\bar{c}_{i}\end{cases}
$$

Thus, if all players have decided to pay their deposit, these deposits are only refunded to those players who contribute to the public good in the previously agreed way, i.e. for whom $c_{i}=\bar{c}_{i}$.

The 2-stage game defined above is denoted by $\widehat{P G}$. Player $i$ 's payoff in $\widehat{P G}$ is given by:

$$
\Pi_{i}\left[\left(d_{1}, c_{1}\right), \ldots,\left(d_{n}, c_{n}\right)\right]=\left\{\begin{array}{l}
\pi_{i}^{*}\left(c_{1}, \ldots, c_{n}\right), \text { if } d_{j}=\bar{d}_{j} \text { for all } j \\
\pi_{i}\left(c_{1}, \ldots, c_{n}\right), \text { else }
\end{array}\right.
$$

A strategy for player $i$ in $\widehat{P G}$ is given by a tuple $\left(d_{i}, \hat{c}_{i}\right)$, where $d_{i} \in\left\{0, \bar{d}_{i}\right\}$ and

$$
\hat{c}_{i}:\left\{0, \bar{d}_{1}\right\} \times \ldots \times\left\{0, \bar{d}_{n}\right\} \rightarrow\left[0, e_{i}\right]
$$

Let $\bar{d}_{i} \leq e_{i}$ be such that

$$
U_{i}\left(e_{i}-\bar{c}_{i}, F\left(\sum_{j \neq i} f_{j}\left(c_{j}\right)+f_{i}\left(\bar{c}_{i}\right)\right)\right)>U_{i}\left(e_{i}-\bar{d}_{i}, F\left(\sum_{j \neq i} f_{j}\left(c_{j}\right)\right)\right)
$$

for all $c_{j} \in\left[0, e_{j}\right], j \neq i$. Since $U_{i}, f_{i}$ and $F$ are strictly increasing and $\bar{c}_{i}>0$, it follows that (2) is satisfied for any $\bar{d}_{i}$ in a sufficiently small neighbourhood of $\bar{c}_{i}$, in particular it is satisfied for $\bar{d}_{i}=\bar{c}_{i}$. The following result, then, is readily established:

Proposition 2 Assume that $\bar{d}_{i}$ satisfies (2) for all $i$. Then, the strategy profile $\left(\bar{d}, \hat{c}^{*}\right)$ where $\bar{d}=\left(\bar{d}_{1} \ldots, \bar{d}_{n}\right)$ and

$$
\hat{c}_{i}^{*}(d)=\left\{\begin{array}{l}
0, \quad \text { if } d_{j}=0 \text { for some } j \\
\bar{c}_{i}, \quad \text { if } d_{j}=\bar{d}_{j} \text { for all } j
\end{array}\right.
$$

is a strict subgame perfect Nash equilibrium of $\widehat{P G}$. Moreover, for all $i, \hat{c}_{i}^{*}$ is 
a strictly dominant strategy at stage 2 and $\bar{d}_{i}$ is a dominant strategy at stage 1 , given that play continues with the strategy profile $\hat{c}^{*}$ at stage 2.

Proof. Consider stage 2 first. If $d_{j}=0$ for some $j$, then $P G$ is played in stage 2. In this case, $\hat{c}_{i}^{*}(d)=0$ for all $i$ is the unique Nash equilibrium and it is in strictly dominant strategies (cf. Proposition 1). If $d_{j}=\bar{d}_{j}$ for all $j$, then $c_{i}=\bar{c}_{i}$ is a strictly dominant strategy for player $i$ in $P G^{*}$. To see this, let $c_{-i}$ be an arbitrary profile of contributions for all players except $i$. By Assumption B it follows that

$$
\begin{aligned}
\pi_{i}^{*}\left(\bar{c}_{i}, c_{-i}\right) & >\pi_{i}^{*}\left(c_{i}, c_{-i}\right) \quad \text { for all } c_{i} \neq \bar{c}_{i} \\
\Longleftrightarrow \pi_{i}^{*}\left(\bar{c}_{i}, c_{-i}\right) & >\pi_{i}^{*}\left(0, c_{-i}\right) \\
\Longleftrightarrow U_{i}\left(e_{i}-\bar{c}_{i}, F\left(\sum_{j \neq i} f_{j}\left(c_{j}\right)+f_{i}\left(\bar{c}_{i}\right)\right)\right) & >U_{i}\left(e_{i}-\bar{d}_{i}, F\left(\sum_{j \neq i} f_{j}\left(c_{j}\right)\right)\right)
\end{aligned}
$$

which is satisfied by definition of $\bar{d}_{i}$. Hence, $\hat{c}_{i}^{*}(d)$ as given in the statement of the proposition is the unique Nash equilibrium in stage 2 and it is in strictly dominant strategies. What remains to be shown is that $\bar{d}_{i}$ is a dominant strategy for player $i$ at stage 1 given that play continues with $\hat{c}^{*}$. Consider first the case where $d_{j}=0$ for some $j \neq i$. Then,

$$
\begin{aligned}
\Pi_{i}\left[\left(\bar{d}_{i}, \hat{c}_{i}^{*}\left(\bar{d}_{i}, d_{-i}\right)\right),\left(d_{-i}, \hat{c}_{-i}^{*}\left(\bar{d}_{i}, d_{-i}\right)\right)\right] & =\pi_{i}\left(\hat{c}^{*}\left(\bar{d}_{i}, d_{-i}\right)\right) \\
& =U_{i}\left(e_{i}, 0\right) \\
& =\pi_{i}\left(\hat{c}^{*}\left(0, d_{-i}\right)\right) \\
& =\Pi_{i}\left[\left(0, \hat{c}_{i}^{*}\left(0, d_{-i}\right)\right),\left(d_{-i}, \hat{c}_{-i}^{*}\left(0, d_{-i}\right)\right)\right]
\end{aligned}
$$


Next, consider the case where $d_{j}=\bar{d}_{j}$, for all $j \neq i$. Then, by (1)

$$
\begin{aligned}
\Pi_{i}\left[\left(\bar{d}_{i}, \hat{c}_{i}^{*}\left(\bar{d}_{i}, \bar{d}_{-i}\right)\right),\left(\bar{d}_{-i}, \hat{c}_{-i}^{*}\left(\bar{d}_{i}, \bar{d}_{-i}\right)\right)\right] & =\pi_{i}^{*}\left(\hat{c}^{*}\left(\bar{d}_{i}, \bar{d}_{-i}\right)\right) \\
& =\pi_{i}^{*}\left(\bar{c}_{1}, \ldots, \bar{c}_{n}\right) \\
& =\pi_{i}\left(\bar{c}_{1}, \ldots, \bar{c}_{n}\right) \\
& >\pi_{i}(0, \ldots, 0) \\
& =\pi_{i}\left(\hat{c}^{*}\left(0, \bar{d}_{-i}\right)\right) \\
& =\Pi_{i}\left[\left(0, \hat{c}_{i}^{*}\left(0, \bar{d}_{-i}\right)\right),\left(\bar{d}_{-i}, \hat{c}_{-i}^{*}\left(0, \bar{d}_{-i}\right)\right)\right]
\end{aligned}
$$

As we have seen in the proof of Proposition 2, for any deposit profile $d$ there is a unique equilibrium $\hat{c}^{*}(d)$ at stage 2 of $\widehat{P G}$. This is not true for stage 1 since $\bar{d}_{i}$ is only weakly dominant for player $i$. Any tuple $\left(d, \hat{c}^{*}\right)$, with $d_{i}=0$ for at least two $i$, also constitutes a Nash equilibrium of $\widehat{P G}$. In such an equilibrium, there is no contribution to the public good at stage 2 . However, $d_{i}=0$ is not a dominant strategy for player $i$, given that play continues with $\hat{c}^{*}$. In fact, $\left(\bar{d}, \hat{c}^{*}\right)$ is the unique subgame perfect Nash equilibrium in stage-wise dominant strategies.

As a last step, we show that the above result remains to hold if the institution collecting and administering the deposits is assumed to be costly, and if players, when paying their deposits, have to bear a small share of this cost. To see this, consider following modification of $\widehat{P G}$, denoted by $\widetilde{P G}$. Different from the previous case, assume now that a fraction $\varepsilon>0$ of any deposit made is kept for the maintenance of the respective institution. Thus, if at the end of stage 1 we have $d_{i}=0$ for some $i$, then in stage 2 the game $P G$ is played except that now player $i$ 's payoff function is given by:

$$
\tilde{\pi}_{i}\left(c_{1}, \ldots, c_{n}\right)= \begin{cases}U_{i}\left(e_{i}-\varepsilon \bar{d}_{i}-c_{i}, F\left(\sum_{j} f_{j}\left(c_{j}\right)\right)\right), & \text { if } d_{i}=\bar{d}_{i} \\ U_{i}\left(e_{i}-c_{i}, F\left(\sum_{j} f_{j}\left(c_{j}\right)\right)\right), & \text { if } d_{i}=0\end{cases}
$$

If at the end of stage 1 we have $d_{i}=\bar{d}_{i}$ for all $i$, then again $P G^{*}$ is played with 
the following modification of the players' payoff functions:

$$
\tilde{\pi}_{i}^{*}\left(c_{1}, \ldots, c_{n}\right)= \begin{cases}U_{i}\left(e_{i}-\bar{d}_{i}-c_{i}, F\left(\sum_{j} f_{j}\left(c_{j}\right)\right)\right), & \text { if } c_{i}<\bar{c}_{i} \\ U_{i}\left(e_{i}-\varepsilon \bar{d}_{i}-c_{i}, F\left(\sum_{j} f_{j}\left(c_{j}\right)\right)\right), & \text { if } c_{i}=\bar{c}_{i}\end{cases}
$$

Hence, player $i$ 's payoff in $\widetilde{P G}$ is

$$
\widetilde{\Pi}_{i}\left[\left(d_{1}, c_{1}\right), \ldots,\left(d_{n}, c_{n}\right)\right]=\left\{\begin{array}{l}
\tilde{\pi}_{i}^{*}\left(c_{1}, \ldots, c_{n}\right), \text { if } d_{j}=\bar{d}_{j} \text { for all } j \\
\tilde{\pi}_{i}\left(c_{1}, \ldots, c_{n}\right), \text { else. }
\end{array}\right.
$$

Proposition 3 below states that also under these modifications everyone paying the deposit in stage 1 and full contributions to the public good in stage 2 still can be implemented as a subgame perfect Nash equilibrium of $\widetilde{P G}$.

Proposition 3 Assume that $\bar{d}_{i}$ satisfies (2) for all $i$. Then, there exists $\bar{\varepsilon}>0$ such that for $0<\varepsilon<\bar{\varepsilon}, \widetilde{P G}$ has exactly two subgame perfect Nash equilibria $\left(d^{*}, \hat{c}^{*}\right)$. In one equilibrium, $d_{i}^{*}=\bar{d}_{i}$ for all $i$ in stage 1 ; in the other equilibrium $d_{i}^{*}=0$ for all $i$ in stage 1 . In both equilibria $\hat{c}^{*}$ is as given in Proposition 2. Both equilibria are strict.

The proof immediately follows from the fact that by continuity inequality (1) implies that

$$
U_{i}\left(e_{i}-\varepsilon \bar{d}_{i}-\bar{c}_{i}, F\left(\sum_{j} f_{j}\left(\bar{c}_{j}\right)\right)\right)>U_{i}\left(e_{i}, 0\right)
$$

and that inequality (2) implies that

$$
U_{i}\left(e_{i}-\varepsilon \bar{d}_{i}-\bar{c}_{i}, F\left(\sum_{j \neq i} f_{j}\left(c_{j}\right)+f_{i}\left(\bar{c}_{i}\right)\right)\right)>U_{i}\left(e_{i}-\bar{d}_{i}, F\left(\sum_{j \neq i} f_{j}\left(c_{j}\right)\right)\right)
$$

for all $i$ if $\varepsilon$ is sufficiently small.

\section{Concluding Remarks}

In this paper, we have proposed a simple two-stage mechanism which implements full contributions to the public good in a subgame perfect Nash equilibrium. 
Similar to a smoker who publicly announces to refrain from smoking in order to make failure prohibitively costly (or a co-author who freely promises to deliver a revised version of the paper by the end of the week), ${ }^{2}$ players in our mechanism can pay an ex ante deposit which, if paid by all, renders contributing to the public good a dominant strategy.

The proposed mechanism, in our view, has at least two favourable properties. First, it implements the provision of public goods in a strict subgame perfect Nash equilibrium. If the cost for running the institution that administers deposits is negligible, the implementation can even be achieved in a stage-wise dominant strategy equilibrium. Hence, even though there exist zero-contribution equilibria as well, the coordination problem is less severe than in case of equilibria that are non-strict or not in dominant strategies. Second, and most importantly, our mechanism does not require the presence or establishment of powerful institutions to implement full contributions to the public good (cf. Falkinger et al., 2000; Fehr and Gächter, 2000; Gürek et al., 2006; Kosfeld et al., 2006). The reason is that no ex post punishment of free-riders is required. All that is needed is an independent institution (e.g. the world bank) that collects deposits, monitors the players' contributions and refunds deposits to those who have contributed to the public good. Hence, our mechanism is particularly suited to implement international agreements like the Kyoto Protocol, where there is no common authority that can enforce the punishment of free-riders.

In many situations, like in the case of global warming, there is a general consensus among the affected parties that the provision of a particular public good is desirable. Yet, at the same time everyone knows that ex post there is a strong incentive to free ride on the contributions of others. Hence, societies who in principle are willing to provide a particular public good (e.g. clean air) can be expected to agree to the implementation of a self-sanctioning scheme (the deposit) which helps them to adhere to their intended contribution ex post. In fact, the mechanism proposed here is indeed quite similar to a self-sanctioning mechanism. Players themselves submit the deposit and, hence, can also easily be assigned the burden of proof that they contributed to the public good. Consequently,

\footnotetext{
${ }^{2}$ Whether announcements are strong enough to later enforce the desired action may be questionable. Their frequent use, however, indicates that the targeted commitment effect indeed is very similar to the one used in our mechanism.
} 
the institution in our case essentially has to administer the deposits, while its monitoring role is rather weak. This is different for a punishing institution, which - in line with conventional legal systems - has to prove that a free-rider did not contribute to the public good.

\section{References}

Bagnoli, M., and B. L. Lipman (1989): "Provision of Public Goods: Fully Implementing the Core through Private Contributions," The Review of Economic Studies, 56, 583-601.

Bosello, F., B. Buchner, And C. Carraro (2003): "Equity, Development, and Climate Change Control," Journal of the European Economic Association, $1,601-611$.

Carraro, C. (1999): International Environmental Agreements on Climate Change. Kluwer Academic Publishers, Dordrecht, The Netherlands.

Clarke, E. H. (1971): "Multipart Pricing of Public Goods," Public Choice, 2, $19-33$.

Dutta, P., And R. Radner (2004): "Self-enforcing Climate Change Treaties," Proceedings of the National Academy of Sciences, 101, 5174-5179.

Falkinger, J., E. Fehr, S. GÄChter, And R. Winter-Ebmer (2000): “A Simple Mechanism for the Efficient Provision of Public Goods: Experimental Evidence," American Economic Review, 90, 247-264.

Fehr, E., And S. GÄChter (2000): "Cooperation and Punishment in Public Goods Experiments," American Economic Review, 90, 980-994.

Green, J., And J. J. Laffont (1979): Incentives in Public Decision Making. North-Holland, Amsterdam.

Groves, T. (1973): "Incentives in Teams," Econometrica, 41, 617-631.

Gürek, O., B. Irlenbusch, And B. Rockenbach (2006): "The Competitive Advantage of Sanctioning Institutions," Science, 312, 108-111. 
JACKSON, M. (1992): "Implementation in Undominated Strategies: A Look at Bounded Mechanisms," Review of Economic Studies, 59, 757-775.

JaCkson, M., And H. Moulin (1992): "Implementing a Public Project and Distributing Its Cost," Journal of Economic Theory, 57, 125-140.

Kosfeld, M., A. Okada, And A. Riedl (2006): "Institution Formation in Public Goods Games," mimeo, Maastricht University.

Moore, J., And R. Repullo (1988): "Subgame Perfect Implementation," Econometrica, 56, 1191-1220.

Palfrey, T., And S. SRIVAStava (1991): "Nash Implementation Using Undominated Strategies," Econometrica, 59, 479-502.

VARIAN, H. R. (1994): "A Solution to the Problem of Externalities When Agents are Well-Informed," The American Economic Review, 84, 1278-1293. 